Volume 14, Number 1, January 2012

\title{
Clinical manifestations and outcomes of severe malaria among children admitted to Rungwe and Kyela district hospitals in south-western Tanzania
}

\author{
AKILI KALINGA ${ }^{1 *}$, MARY MAYIGE ${ }^{1}$, GIBSON KAGARUKI ${ }^{1}$, AMANI SHAO ${ }^{1}$, BRIGHTON \\ MWAKYUSA $^{1}$, FRANK JACOB ${ }^{2}$ and CHARLES MWESIGA ${ }^{3}$ \\ ${ }^{1}$ Tukuyu Medical Research Centre, P.O. Box 538, Tukuyu, Tanzania \\ ${ }^{2}$ Rungwe District Council, P.O. Box 148, Tukuyu, Tanzania \\ ${ }^{3}$ Kyela District Council, P.O. Box 4, Tukuyu, Tanzania
}

\begin{abstract}
Malaria remains as an important public health and a major cause of childhood death and paediatric hospital admission in sub-Saharan Africa. This prospective hospital based cross sectional study was conducted from April 2007 to April 2008. The main objective was to assess clinical manifestations and outcomes of severe malaria in children admitted to district hospital in Rungwe and Kyela in south-western Tanzania. A total of 1371 children were selected as screening group of which 409 (29.8\%) were tested positive for malaria. Mean age of the children was 2.7 $(95 \% \mathrm{CI}=2.5,2.8)$ years and the majority $(86 \%)$ were under five years of age. The proportion of children severe malaria in Rungwe was significantly higher than that of Kyela by $21.3 \%(P=0.002)$. The common symptoms of severe malaria during admission were convulsions $(50.9 \%)$ compensated shock $(30.6 \%)$, prostration $(29.1 \%)$ and symptomatic severe anaemia $(14.9 \%)$. The case fatality rate (CFR) was $4.6 \%$ and the cure rate (CR) was $95.4 \%$. Children with suspected severe acidosis and symptomatic severe anemia were 4.8 (95\% CI=1.6, 14.6) and 5.5 (95\%CI 1.1, 28.2), respectively, more likely to die compared to those without these symptoms. The proportion of deaths among children presenting $\geq 5$ symptoms was $32.1 \%$ higher than among those presenting one symptom ( $\mathrm{OR}=0.50,95 \% \mathrm{CI} 0.125-2.000 ; P=0.000)$. Convulsions and compensated shock were the leading symptoms at admission. Suspected severe acidosis and symptomatic severe anemia were the predictors of mortality for children. In order to reduce mortality among admitted children with severe malaria there is a need for health providers to deploy strategic management of fatal prognostic factors. In conclusion, convulsion and compensated shock were the leading symptoms among children at admission and that suspected severe acidosis and symptomatic severe anemia were the predictors of mortality. It is therefore important to emphasis early diagnosis and prompt treatment of severe cases of malaria to minimize mortality among children.
\end{abstract}

Keywords: malaria, children, hospital, clinical, symptoms, outcome, Tanzania

\section{Introduction}

Malaria remains a leading cause of morbidity and mortality especially among children and pregnant women in sub-Saharan Africa where at least $90 \%$ of malaria deaths occur (WHO, 2005). The World Health Organization estimates that the number of malaria deaths in young children in sub-Saharan Africa in 2000 ranged from 710,000 to 896,000 (WHO \& UNICEF, 2003). It is generally understood that malaria causes around $20 \%$ of

\footnotetext{
* Correspondence: Akili K. Kalinga; E-mail: kalingaaka@yahoo.com/akalinga@nimr.or.tz
} 
all deaths in children under 5 years old in Africa and that it is now the most important cause of death in this group. In Tanzania there are an estimated 14-19 million cases of malaria annually and malaria is reportedly the cause of one third of all outpatient visits and hospital admissions. It is the main cause of admission for children (38\%) and adults $(32 \%)$ in health facilities (Rugemalila et al., 2006). In Tanzania, malaria accounts for an estimated 16 million clinical episodes and 120,000 deaths occur per year and that of these deaths, over 58\% are in children less than five years of age (Makundi et al., 2007).

Severe malaria is defined as history of fever plus at least one symptom of severe malaria and positive malaria test (WHO, 2010). Severe malaria mainly affects children under 5 years old, non-immune travellers, migrants to malarial areas, and people living in areas with unstable or seasonal malaria (Sheehy \& Angus, 2011). In endemic countries with a high transmission of malaria, severe malaria is predominantly a disease of young children of 1 month to 5 years of age (Trampuz et al., 2003). Criteria for severe malaria have been established to assist clinical and epidemiological studies (WHO, 1990, 2000). The symptoms of severe malaria includes prostration, altered consciousness, lethargy or coma, respiratory distress, severe anaemia, convulsions, inability to swallow, persistent vomiting and dark or limited urine for adults only (http://archives.who.int/eml/expcom/expcom15/applications/formulations/artesunate.pd f). Studies in Ghana and Gabon reported that severe anemia, prostration, respiratory distress, convulsions, impaired consciousness and hyperlactaemia to be the major symptoms of severe malaria (Mockenhaupt et al., 2004; Dzeing-Ella et al., 2005).

The major complications of severe malaria include cerebral malaria, pulmonary oedema, acute renal failure, severe anemia, and/or bleeding. Acidosis and hypoglycemia are the most common metabolic complications. Any of these complications can develop rapidly and progress to death within hours or days (WHO, 2000). In clinical practice, patients must be assessed for any of these signs or symptoms that suggest an increased risk for developing complications and must be treated immediately.

Optimal care for severe malaria requires well-developed diagnostic facilities and intensive care. Centralizing these capabilities may be logical in a managerial sense but will not benefit the people at greatest risk. It is therefore, important to enable peripheral health services to provide emergency treatment by means of guidelines, training, and the regular supply of medicines and diagnostic supplies and equipment (FMoH, 2004). At the periphery, the priority requirement is the rapid recognition of the signs and symptoms of severe malaria that should lead to emergency care or referral to a higher level of care. It was therefore, the objective of this study to assess clinical manifestations and outcomes of severe malaria in children admitted to district hospital in Rungwe and Kyela in south-western Tanzania.

\section{Methods and Materials}

\section{Study area}

This hospital based study was done in Kyela and Rungwe districts in south-western Tanzania. Rungwe district has a highly variable terrain, while Kyela is mainly formed of 
low-lying flood plains on the northern shores of Lake Malawi, at $475 \mathrm{~m}$ altitude. To its southern borders, Rungwe district shares a small strip of lowland with Kyela, but its altitude rises within a relatively short distance towards the north, northeast and northwest, to the volcanic summits of Mt. Rungwe and Kyejo, which rise to above $4,000 \mathrm{~m}$. The two districts have an estimated population of 500,000.

\section{Study population and sampling technique}

The study population was children with severe malaria admitted in the paediatric wards of the district hospitals in Kyela and Rungwe. Inclusion criteria were a child clinically diagnosed to have history of fever, at least one symptom of severe malaria, tested positive for malaria and aged 1 month to 12 years. The exclusion criteria were any child aged below one month and those above 12 years of age, clinically diagnosed to have no fever, not having any symptom of severe malaria and tested negative for malaria.

Paediatric wards in Kyela and Rungwe district hospitals were purposively selected. Although the target screening group of children was 1400, a total of 1371 children clinically diagnosed to have history of fever and at least one symptom of severe malaria were obtained out of 5,753 children admitted from April 2007 to April 2008. Of the children having history of fever and meeting at least one of the WHO 2000 malaria severity criteria, 440 children inclusive $10 \%$ for non-response were anticipated to be recruited for study upon being confirmed to have severe malaria from blood smear microscopy but the team recruited 409 in 24 hours every day.

\section{Clinical procedures and laboratory tests}

Children admitted to the paediatric wards were examined for symptoms or signs of malaria by either nurses, clinical officers, assistant medical officers or medical officers to ascertain if the child had history of fever and any of the symptoms of severe malaria. Indicators for hypoglycemia, shock were determined using WHO guidelines. The following are symptoms and their specifications as were considered for screening to participate in the study: Coma (Glasgow Coma Scale (GCS) $\leq 10$, Blantyre Coma Scale (BCS) $\leq 2$ ), prostration (not able to breastfed $<6 \mathrm{~m}$, or able to sit $>6 \mathrm{~m}$ ), convulsions ( $>30$ minutes), decompensated shock (children: systolic blood pressure $<70 \mathrm{mmHg}$ ), compensated shock (capillary refill $\geq 3 \mathrm{sec}$. /temperature gradient) and severe respiratory distress (nasal flaring, lower chest in drawing/retractions). Other symptoms for qualification for enrolment group were either a child with suspected severe acidosisdeep breathing, hypoglycemia (BM-Stix $<3 \mathrm{mmol} / \mathrm{L}$ or clinical improvement after IV glucose), symptomatic severe anaemia $(\mathrm{Hb}<5 \mathrm{~g} / \mathrm{dl})$ in combination with respiratory distress), black water fever, severe jaundice and anuria in older children.

Thick peripheral blood smears were performed to detect Plasmodium falciparum infection. Parasites were counted against 200 white blood cells. For recruited patients, absence of malaria after treatment was declared after 3 consecutive negative blood smears at 8 hourly intervals. Haemoglobin level was tested with a colorimeter using Drabkin's method and blood sugar glucose was tested using Glucometer machine. 


\section{Data analysis}

Data was entered on Epidata version 3.1 and later exported to SPSS Version and analysed by Stata version 10. Chi-square test was performed to measure any association between independent and dependent variables. Unadjusted and logistics (adjusted) analysis was done to assess the relationships between the dependent and independent variables. The difference in means was tested using t-test. Statistical difference and associations between variables was tested at 5\% significance level.

\section{Ethical considerations}

Ethical clearance was obtained from National Ethical Review Committee of the Medical Research Coordination Committee of the National Institute for Medical Research. Permission to conduct research in paediatric wards was sought from District Medical Officers, in charges of hospitals and matron of the respective wards. Oral consents were obtained from children's parents or caretakers.

\section{Results}

A total of 1371 children were included in the study. Of these, 409 (29.8\%) children tested positive for malaria and hence confirmed to have severe malaria. Of the confirmed cases, $39.4 \%$ were from Kyela and 60.6\% from Rungwe. Males constituted 53\% while female accounted for $47 \%$ of the children. Mean age of the children was $2.7(95 \% \mathrm{CI}=2.5,2.8)$ years. Majority $(86 \%)$ of the children were under five years of age (Kyela=91.9\%; Rungwe $=82.3 \%$ ). The proportion of children severe malaria in Rungwe was significantly higher than that of Kyela by $21.3 \%(P=002)$.

Table 1: Common symptoms of severe malaria by confirmed patients $(n=409)$

\begin{tabular}{|c|c|c|c|c|c|c|c|}
\hline \multirow[t]{2}{*}{ Symptom } & \multirow{2}{*}{$\begin{array}{c}\text { Overall } \\
\text { cases }\end{array}$} & \multirow[t]{2}{*}{$\%$} & \multicolumn{2}{|c|}{ Kyela } & \multicolumn{2}{|c|}{ Rungwe } & \multirow[t]{2}{*}{ P-value } \\
\hline & & & Freq & $\%$ & Freq & $\%$ & \\
\hline Coma & 40 & 9.8 & 18 & 45.0 & 22 & 55.0 & 0.273 \\
\hline Prostration & 119 & 29.1 & 40 & 33.6 & 79 & 66.4 & 0.127 \\
\hline Convulsions & 208 & 50.9 & 74 & 35.6 & 134 & 64.4 & 0.068 \\
\hline Decompensated shock & 33 & 8.1 & 6 & 18.2 & 27 & 81.8 & 0.006 \\
\hline Compensated shock & 125 & 30.6 & 92 & 42.0 & 125 & 57.6 & 0.109 \\
\hline Severe respiratory distress & 23 & 5.6 & 13 & 56.5 & 10 & 43.5 & 0.066 \\
\hline Severe acidosis & 14 & 3.4 & 8 & 57.1 & 6 & 42.9 & 0.135 \\
\hline Hypoglycemia & 8 & 2.0 & 4 & 50.0 & 4 & 50.0 & 0.390 \\
\hline Severe anaemia & 58 & 14.2 & 22 & 37.9 & 36 & 62.1 & 0.465 \\
\hline Black water fever & 8 & 2.0 & 1 & 12.5 & 7 & 87.5 & 0.111 \\
\hline Severe jaundice & 5 & 1.2 & 0 & 0.0 & 5 & 100.0 & - \\
\hline Anuria / oliguria & 0 & 0.0 & 0 & 0.0 & 0 & 0.0 & _ \\
\hline Hyperparasitaemia & 25 & 6.1 & 2 & 7.4 & 25 & 92.6 & 0.000 \\
\hline
\end{tabular}

Note: Multiple responses 
During the period of study (390 days) the mean daily admission was $0.52(0.46,0.59)$ person per day. It was higher in Rungwe $(95 \% \mathrm{CI}=0.54,0.73)$ than in Kyela $(95 \% \mathrm{CI}=0.33$, 0.49) $(P=0.001)$. The common symptoms of severe malaria were convulsions $(50.9 \%)$ compensated shock $(30.6 \%)$, prostration $(29.1 \%)$ and symptomatic severe anaemia $(14.9 \%)$. There was significant variation in presentation of symptoms of severe malaria between the two hospitals. The frequency of hyperparasitaemia (92.6\%) and decompasated shock (81.8\%) were higher in Rungwe than Kyela (Table1). The majority (75- $92.6 \%$ ) of symptoms of severe malaria were observed among $<5$ years children than in $\geq 5$ years $(7.4-25 \%$ ). The overall case fatality rate (CFR) was $4.6 \%$ (Kyela=7.5\%; Rungwe $=2.8 \%$ ). The proportion of deaths among children in Kyela was higher than in Rungwe by $23.6 \%$. There was no significance difference in death rates between $<5$ years and $\geq 5$ years children or between females and males.

Table 2: Unadjusted odds ratio (or) for the symptoms of severe malaria on death

\begin{tabular}{lllll}
\hline Symptom & OR & $\mathbf{X}^{\mathbf{2}}$ & $95 \% \mathrm{CI}$ & P-value \\
\hline Coma & 4.830 & 10.700 & $1.700,13.70$ & 0.001 \\
Prostration & 1.450 & 0.580 & $0.550,3.780$ & 0.447 \\
Convulsions & 1.350 & 0.390 & $0.523,3.420$ & 0.530 \\
Decompensated shock & 3.310 & 4.520 & $1.030,10.740$ & 0.033 \\
Compensated shock & 0.980 & 0.000 & $0.390,2.470$ & 0.970 \\
Severe respiratory distress & 2.067 & 0.900 & $0.446,9.578$ & 0.343 \\
Suspected severe acidosis & 6.460 & 9.200 & $1.611,25.899$ & 0.002 \\
Hypoglycaemia & 7.529 & 7.610 & $1.389,40.818$ & 0.006 \\
Symptomatic severe anaemia & 3.877 & 8.390 & $1.442,10.424$ & 0.004 \\
Black water fever & 3.040 & 1.130 & $0.352,26.189$ & 0.287 \\
Hyperparasitaemia & 1.717 & 0.500 & $0.374,7.877$ & 0.481 \\
\hline
\end{tabular}

Note: Multiple responses

Table 3: Adjusted odds ratio (OR) analysis for the symptoms of severe malaria on death

\begin{tabular}{lcccc}
\hline Symptom & OR & Z & 95\%CI & P-value \\
\hline Coma & 2.74 & 1.48 & $0.72,10.44$ & 0.140 \\
Prostration & 0.95 & -0.09 & $0.29,3.14$ & 0.929 \\
Convulsions & 1.92 & 1.17 & $0.64,5.74$ & 0.244 \\
Decompensated shock & 2.24 & 1.09 & $0.53,9.51$ & 0.275 \\
Compensated shock & 1.91 & 1.14 & $0.63,5.76$ & 0.253 \\
Severe respiratory distress & 1.64 & 0.56 & $0.28,9.54$ & 0.584 \\
Suspected severe acidosis & 5.47 & 2.03 & $1.06,28.22$ & 0.043 \\
Hypoglycaemia & 6.43 & 1.76 & $0.81,51.13$ & 0.078 \\
Symptomatic severe anaemia & 4.81 & 2.78 & $1.59,14.55$ & 0.005 \\
Black water fever & 1.95 & 0.51 & $0.15,24.87$ & 0.608 \\
Hyperparasitaemia & 1.39 & 0.39 & $0.27,7.34$ & 0.695 \\
\hline
\end{tabular}

Note: Multiple responses 
With unadjusted odds ratio analysis five out of 13 symptoms showed statistical significance on the odds of dying. At $95 \% \mathrm{CI}$, the risk of death was associated with coma $(95 \% \mathrm{CI}=1.7,13.7)$, decompensated shock $(95 \% \mathrm{CI}=1.0,10.7)$, suspected severe acidosis $(95 \% \mathrm{CI}=1.6,25.9)$, hypoglycaemia $(1.4,40.8)$ and symptomatic severe anaemia $(95 \% \mathrm{CI}$ $=1.4,10.4$ ) (Table 3). However, using logistics analysis for adjusted odds ratio only severe acidosis $(95 \% \mathrm{CI}=1.1,28.2)$ and symptomatic severe anaemia (95\% $\mathrm{CI}=1.6,14.6)$ were associated with high risk of death (Table 3). The probability of dying from severe malaria increased with an increase in the number severe symptoms the child presented at admission (Table 4). The proportion of deaths among children who presented five and more symptoms was $33.3 \%$ and those presented with four symptoms was $13.8 \%$ compared to those presented with one symptom (1.3\%).

Table 4: Number of symptoms at admission on children' outcomes

\begin{tabular}{ccccccccc}
\hline \multirow{2}{*}{$\begin{array}{c}\text { No. of } \\
\text { Symptoms }\end{array}$} & Admissions & \multicolumn{2}{c}{ Survived } & \multicolumn{2}{c}{ Deaths } & Odds & 95\% CI & P-value \\
\cline { 2 - 6 } & & Freq & $\mathbf{\%}$ & Freq & $\mathbf{\%}$ & & & \\
\hline 1 & 156 & 154 & 98.7 & 2 & 1.3 & 0.013 & $0.003,0.052$ & 0.000 \\
2 & 170 & 163 & 95.9 & 7 & 4.1 & 0.043 & $0.020,0.091$ & \\
3 & 45 & 42 & 93.3 & 3 & 6.7 & 0.071 & $0.022,0.230$ & \\
4 & 29 & 25 & 86.2 & 4 & 13.8 & 0.160 & $0.055,0.459$ & \\
$5+$ & 9 & 6 & 66.7 & 3 & 33.3 & 0.500 & $0.125,2.00$ & \\
Total & $\mathbf{4 0 9}$ & $\mathbf{3 9 0}$ & $\mathbf{9 5 . 6}$ & $\mathbf{1 9}$ & $\mathbf{4 . 6}$ & - & - & \\
\hline
\end{tabular}

\section{Discussion}

Findings from this study showed that about third of the screened children were diagnosed to have severe malaria. The proportion of children with severe malaria in Rungwe district was found to be significantly higher than that of Kyela by $21.3 \%$. In our opinion, the difference can be due to the fact that children in Rungwe district which is located in high attitude (up to about $4000 \mathrm{~m}$ a.s.l.) were not immune enough to malaria infection compared to those in Kyela which is endemic to malaria. Therefore, children in Rungwe district within area of unstable or seasonal malaria were likely to be severely ill from infection than those from Kyela district. The study also revealed that many children with severe malaria had either one or a combination of symptoms. Convulsion was observed to be the most common symptom of severe malaria among children. The frequency of convulsions among children in our study was higher than that reported in Ghana (Mockenhaupt et al, 2004) and Uganda (Idro \& Aloyo, 2004). However, the proportion of children with convulsion in our study was similar to that found in a study done in Senegal (Camara et al, 2011).

Although in several studies (Maitland et al., 2003; Idro \& Aloyo, 2004) anaemia has been reported as a common symptom of severe malaria, in this study only a small proportion of the children (14.2\%) presented with severe anaemia. The reasons for low prevalence of anaemia among severe cases of malaria could not be established. This calls for further studies to ascertain the ambiguity. 
The case fatality rate $\mathrm{n}$ this study is lower that reported elsewhere in Africa (Mockenhaupt et al., 2004; Dondorp et al., 2010). However, the low CFR observed in our study is in agreement with studies carried out in Uganda (Idro \& Aloyo, 2004) and in Gabon (Issifou et al., 2007). Interestingly, in a study done among children admitted to Kumasi Hospital in Ghana, none of the children with severe malaria died (Gyapong et al., 2009). The low CFR in our study is likely be attributed to early treatment seeking behaviour among children care seekers and good management of severe malaria.

Although in this study, severe acidosis and symptomatic severe anaemia were major predictors of death, in Kenya, Gabon and Senegal, shock, hyperlactaemia, hypoglycaemia, respiratory distress, impaired consciousness, and jaundice were associated with larger proportions of all deaths (Marsh et al., 1995; Maitland et al., 2003; Dzeing-Ella et al., 2005; Issifou et al., 2007). In India, Tripathy et al. ( 2007) reported respiratory distress, coma, multiple organ dysfunctions, and hyperparasitemia as major predictors of death. Unlike in our study, a study in Ghana reported severe anaemia as the leading manifestation of severe malaria (Mockenhaupt et al., 2004). In a study in Senegal young age was also identified as one of the main death risk factors (Camara et al., 2011).

It has been observed that the number of symptoms the child present has a significant influence on the treatment outcomes. As the number of symptoms increases, the probability of a child to die also increases. That means the more the number of complications the child had, the difficult in its management. This finding is in agreement with the observations in Gabon (Dzeing-Ella et al., 2005) and India (Tripathy et al., 2007)

In conclusion, convulsion and compensated shock were the leading symptoms among children at admission and that suspected severe acidosis and symptomatic severe anemia were the predictors of mortality. Moreover, the probability of a child to die increased with increased number of symptoms of severe malaria a child had during admission. In order to reduce mortality among children with severe malaria, it is important that health need to invest more efforts in promoting community early identification of malaria symptoms and presentation to health care facilities for appropriate management of severe malaria.

\section{References}

Camara, B., Diagne-Gueye, N.R., Faye, P.M., Fall, M.L., Ndiaye, J.L., Ba, M. \& Sow, H.D. (2011) Malaria severity criteria and prognostic factors among children in Dakar. Médecine et Maladies Infectieuses 41, 63-67.

Dondorp, A.M., Fanello, C.L., Hendricksen, L.C.E., Gomes, E., Seni, A., Chhaganlal, K.D., Bojang, K., Olaosebikan,R., Anunobi,N., Maitland, K., Kivaya, E., Agbenyega,T., Nguah, S.B. Evans,J., Gesase,S., Kahabuka, C., Mtove, G., Nadjm,B., Deen, J., Mwanga-Amumpaire,J., Nansumba, M., Karema,C., Umulisa, N., Uwimana, A., Mokuolu, O.A., Adedoyin, O.T., Johnson, W.B.R., Tshefu, A.K., Onyamboko, M.A., Sakulthaew,T., Ngum, W.P., Silamut, K., Kasia Stepniewska, K., Woodrow, C.J., Bethell, D., Wills, B., Oneko, M., Petom, 
T.E., Seidlein, L.V., Day, N.PJ., White, N.J. \& for the AQUAMAT group (2010) Artesunate versus quinine in the treatment of severe falciparum malaria in African children (AQUAMAT): an open-label randomized trial. Lancet 376, 16471657

Dzeing-Ella, A., Nze Obiang, P.C., Tchoua, R., Planche, T., Mboza,B., Mbounja, M., Muller-Roemer, U., Jarvis, J., Kendjo, E., Ngou-Milama, E., Kremsner, P.G., Krishna, S. \& Kombila, M. (2005) Severe falciparum malaria in Gabonese children: clinical and laboratory features Malaria Journal 4:1, 167-172.

FMoH (2004) Malaria Diagnosis and Treatment Guidelines for Health Workers in Ethiopia Addis Ababa, Ethiopia

Gyapong, R.N.K., Duwiejua, M., Bio, F.Y., Woode, E., Ansah, C., Owusu-Daaku, F.T. \& Buabeng, K.O. (2009) Characterization and Treatment of Severe Malaria in Hospitalized Children at a Ghanaian District Hospital. The Open Tropical Medicine Journal 2, 39-44.

Idro, R. \& Aloyo, J. (2004) manifestations, quality of emergency care and outcome of severe malaria in mulago hospital, Uganda. African Health Sciences 4, 50-57.

Issifou, S., Kendjo, E., Missinou,M., Matsiegui, P., Dzeing-Ella, A., Dissanami,F., Kombila,M., Krishna, S. \& Kremsner, P. (2007) differences in presentation of severe malaria in urban and rural Gabon. American Journal of Tropical Medicine Hygiene 77, 1015-1019.

Maitland, K., Levin, M., English, M., Mithwani, S., Peshu, N., Marsh, K. \& Newton, C.R. (2003) Severe P. falciparum malaria in Kenyan children: evidence for hypovolaemia. International Journal of Medicine (QJM) 96, 427-434.

Makundi, E.A., Malebo, H.M., Mhane, P., Kitua, A.Y. \& Warsame, M. (2006) Role of traditional healers in the management of severe malaria among children below five years of age: the case of Kilosa and Handeni districts, Tanzania. Malaria Journal 5:58.

Marsh, K., Forster, D., Gaiuru, C., Mwangi, I., Winstanley, M., Marsh, V., Newton, C., Winstanley, P., Warn, P., Peshu, N., Pasvol, G. \& Snow, R.(1995) Indicators of life-threatening malaria in African children. New England Journal of Medicine 332, 1399-1404.

Mockenhaupt, F.P., Ehrhardt, S., Burkhardt, J., Bosomtwe, S.Y., Laryea, S., Anemana, S.D., Otchwemah, R.N., Cramer, J.P., Dietz, E., Gellert, S. \& Bienzle, U. (2004) Manifestation and outcome of severe malaria in children in northern Ghana. American Journal of Tropical Medicine Hygiene 71, 167-172.

Rugemalila, J., Wanga, C. \& Kilama, W. (2006) Sixth Africa malaria day in 2006: how far have we come after the Abuja Declaration? Malaria Journal 5:102.

Sheehy, S.H. \& Angus, B.J. (2011) Malaria: severe, life-threatening. Clinical Evidence pii: 0913.

Trampuz, A., Jereb, M., Muzlovic, I. \& Prabhu, R.M. (2003) Clinical review: Severe malaria. Critical Care 7, 315-323.

Tripathy, R., Parida, S., Das, L., Mishra, D., Tripathy, D., Das, M., Chen, H., Maguire, J. \& Panigrahi, P. (2007) Clinical Manifestations and Predictors of 
Severe Malaria in Indian Children. Pediatrics 120, 3 e454-e460.

WHO (2010) Guidelines for the Treatment of Malaria - $2^{\text {nd }}$ Edition. World Health Organization, Geneva.

WHO (2005) Malaria Control Today: Current WHO Recommendations. World Health Organization, Geneva, Working Document [www.who.int/malaria]

WHO (2000) New Perspectives: Malaria Diagnosis. Report of a Joint WHO/USAID: Informal Consultation held on 25-27 October 1999. Geneva, Switzerland.

WHO (2000) Severe falciparum malaria. Transactions of Royal Society of Tropical Medicine Hygiene 94 (Suppl 1), S1-S90.

WHO (1990) Severe and complicated malaria. Transactions of Royal Society of Tropical Medicine Hygiene 84 (Suppl 2), S1-S65.

WHO (undated document) Artesunate for the treatment of severe malaria: global malaria programme. World health organization, Geneva, Switzerland (Available at http://archives.who.int/eml/expcom/expcom15/applications/formulations/artesu nate.pdf visited on 10/05/2011).

WHO \& UNICEF (2003) The Africa Malaria Report. WHO/CDS/MAL/2003.1093. 